\title{
Linx
}

Revue des linguistes de l'université Paris X Nanterre

$72 \mid 2015$

Former à l'écrit universitaire, un terrain pour la linguistique?

\section{La compétence linguistique (acquisition et enseignement) dans la formation à l'écriture universitaire en FLE/FLS : étude de cas}

\section{Catherine Carras}

\section{(2) OpenEdition}

\section{Journals}

Édition électronique

URL : http://journals.openedition.org/linx/1607

DOI : 10.4000/linx.1607

ISSN : 2118-9692

Éditeur

Presses universitaires de Paris Nanterre

Édition imprimée

Date de publication : 1 septembre 2015

Pagination : 73-94

ISSN : 0246-8743

Référence électronique

Catherine Carras, «La compétence linguistique (acquisition et enseignement) dans la formation à l'écriture universitaire en FLE/FLS : étude de cas », Linx [En ligne], 72 | 2015, mis en ligne le 01 mars 2016, consulté le 10 décembre 2020. URL : http://journals.openedition.org/linx/1607 ; DOI : https:// doi.org/10.4000/linx.1607 


\section{La compétence linguistique (acquisition et enseignement) dans la formation à l'écriture universitaire en FLE/FLS : étude de cas \\ Catherine CARRAS}

Laboratoire Lidilem, Université Stendhal Grenoble 3

\section{Résumé}

Cet article s'interroge sur la place de la compétence linguistique dans la formation à l'écriture universitaire pour les publics FLE/FLS. Pour ce faire, nous étudierons un dispositif de formation à l'écrit universitaire mis en place pour des doctorants algériens. Du point de vue de l'apprentissage, nous nous interrogerons sur les compétences linguistiques qui peuvent être considérées comme insuffisantes, à travers l'étude des énoncés considérés comme déviants dans les productions écrites du public observé: quelles parties du discours sont concernées ? Quelle influence ces énoncés déviants ont-ils sur la cohérence du discours dans son ensemble? Du point de vue de l'enseignement, nous analyserons le dispositif de formation mis en place pour ce public, en particulier la place occupée par le travail sur les compétences linguistiques. La question sous-jacente étant: comment sortir du microlinguistique, tant dans la gestion des énoncés considérés comme déviants, que dans les remédiations proposées?

Mots-clés

Didactique du FLE, Français sur Objectif Universitaire, littératie universitaire

\section{Compétences linguistiques et littératie universitaire}

La problématique des littératies universitaires a connu lors de la dernière décennie un développement remarquable, tant du côté de la recherche fondamentale que de ses applications pratiques, dans la formation à l'écrit universitaire des étudiants francophones et non francophones.

La notion de littératie universitaire, "structurée autour d'une critique ethnographique et socioculturelle des pratiques académiques dominantes et de la conception traditionnelle de l'écriture comme ensemble d'habilités techniques » (Delcambre et Lahanier-Reuter, 2012:8), considère la pratique de l'écrit à l'université de façon globale, en tant que pratique sociale et culturelle sur le terrain spécifique que représente l'université. C'est pour cela que nous préférons cette notion à celle, plus restrictive et qui pourtant correspond assez bien au travail que nous effectuons auprès des publics d'étudiants non francophones, de «didactique du français à l'université », qui se réfère à des pratiques d'enseignement du français comme langue ou à des pratiques de remédiation des difficultés d'expression écrite des étudiants (idem : 9). En effet, la notion de littératie en tant que pratique sociale et culturelle permet de considérer les besoins des étudiants non seulement en termes de formation mais de façon plus générale en termes d'acculturation. La compétence en langue apparaît donc comme un facteur parmi d'autres dans l'acculturation aux discours universitaires (Pollet, 2001).

Or, on remarque que les dispositifs de mesure des capacités langagières des étudiants (tests lors de l'entrée à l'université, entre autres), les diagnostics sur la maîtrise de la littératie chez les étudiants de la part des enseignants, et par conséquent les dispositifs de remédiation aux éventuels déficits constatés sont le plus souvent centrés sur des compétences linguistiques ponctuelles et normatives (Mortamet, 2002 ; Delbart, 2002). Cette remarque ne constitue en rien un jugement de valeur, il est clair que les erreurs de type linguistique dans les discours des étudiants sont plus visibles et donc plus facilement diagnosticables. Néanmoins, cette 
centration sur le linguistique, et même sur le micro-linguistique, pose un certain nombre de questions.

En effet, cette centration relève d'une conception instrumentale de la langue, qui soumettrait la maîtrise de la langue de l'écrit à celle de micro-habiletés linguistiques (Laborde-Milaa, Boch et Reuter, 2004 : 6), alors que la maîtrise de l'écrit n'est évidemment pas la résultante mécanique de l'apprentissage de l'orthographe, des temps verbaux, etc. (Carton, 2002 : 163). La focalisation sur les formes linguistiques peut occulter les problèmes de haut niveau de l'écriture, comme le rapport au savoir, les problèmes pragmatiques et textuels (idem), et le concept de littératie universitaire prône justement une prise en compte globale des compétences liées à l'écrit.

Et pourtant ... si de multiples facteurs entrent en jeu dans les difficultés actuelles des étudiants face à l'écrit, on ne peut nier que des lacunes au niveau de la maîtrise de la langue en font partie. Dans les formations à l'écrit universitaire destinées aux étudiants non francophones, en particulier, on peut constater que la question de la langue et de la formation linguistique est bien présente. En effet, une «montée en compétence » au niveau linguistique chez ce type de public est indispensable, car si les dispositifs destinés à des étudiants francophones sont surtout axés sur la remédiation, en FLE et FLS il s'agit avant tout de perfectionnement voire d'acquisition de compétences linguistiques. Ainsi, nous suivons Delcambre et Lahanier-Reuter (2010) qui soulignent que l'on ne doit pas considérer les difficultés des étudiants face aux écrits universitaires uniquement en termes de «déficit linguistique »; si ceci est vrai pour les publics francophones natifs, ce l'est encore plus pour les publics non natifs, on ne peut qualifier de « déficit» ce besoin de formation linguistique qui est une caractéristique inhérente à ces publics. Il faut ainsi reconnaître et prendre en charge ce besoin de formation linguistique, même si, dans le cadre de la notion de littératie universitaire, il convient de ne pas isoler le travail sur les compétences linguistiques. Toute la difficulté réside donc dans la nécessité de prendre en charge ce besoin de formation linguistique, tout en incluant cette formation dans une compétence plus large de maîtrise de l'écrit. Cette problématique nous paraît centrale pour une véritable prise en charge des difficultés des publics non francophones, très présents dans les filières de nos universités, et avec des niveaux de langue parfois très faibles, en particulier dans les filières scientifiques.

En effet, ce que nous constatons le plus souvent dans les formations à la «Méthodologie Universitaire ${ }^{1}$ qui se mettent en place de plus en plus fréquemment à destination des publics non francophones, en particulier ceux inscrits en Master ou Doctorat, c'est un cloisonnement entre le travail sur les compétences linguistiques et celui sur les aspects plus méthodologiques. Les cours de langue proprement dits sont souvent pris en charge par les centres de Français Langue Etrangère des Universités, en amont, et les cours de méthodologie plutôt par les Ecoles Doctorales ou les UFR. Il manque à notre avis, dans la prise en charge de ces publics, une véritable collaboration entre enseignants de langue et enseignants de disciplines. Ce cloisonnement souvent constaté entre formation linguistique et formation méthodologique aboutit à une «fracture » dans la prise en charge des difficultés de ces publics: les cours de langue sont le plus souvent des cours généralistes (même si, en particulier dans le cas des publics de faible niveau, nous ne nions pas le besoin d'acquisition de compétences linguistiques de base), qui ne ciblent pas les discours universitaires et leurs spécificités, et les cours de méthodologie ne prennent que rarement en compte les difficultés purement linguistiques de ces étudiants.

\footnotetext{
${ }^{1}$ Nous utilisons cette appellation générale de «Méthodologie Universitaire» sachant qu'elle recouvre des dispositifs de formation qui diffèrent suivant les institutions et les publics.
} 
C'est à notre avis du côté du Français sur Objectif Universitaire (Mangiante et Parpette, 2010) et de sa méthodologie que se trouve la démarche la plus globale de prise en charge des publics non francophones et de leurs difficultés. L'objectif final est certes celui de la maîtrise des discours universitaires, mais c'est la langue, le français, qui en est le point d'entrée, et d'ancrage. Ainsi, comme le soulignent ces auteurs « une production écrite, même au sein d'un domaine scientifique très spécialisé, demeure une production linguistique répondant aux mêmes règles et conditions que celles de tout texte » (Mangiante et Parpette, 2010 : 148). Des exemples d'analyse de productions écrites d'étudiants non francophones figurent dans cet ouvrage et les constatations des auteurs sont que les difficultés des étudiants ne portent pas forcément sur le domaine de spécialité ni même, le plus souvent, sur le lexique spécialisé, mais bien sur la maîtrise imparfaite de la syntaxe, des articulateurs et connecteurs logiques, de la conjugaison, qui constituent des éléments de cohésion et de structuration du texte. Et c'est justement pour faire acquérir ces compétences langagières requises qu'intervient l'enseignant de langue.

\section{Objectifs et corpus d'observation}

Nous souhaitons donc, dans cet article, «porter un regard de didacticien du français sur la littératie universitaire » (Ronveaux, 2010: 158) (et nous ajouterons, un regard de didacticienne du Français Langue Etrangère) et nous attacher à la part linguistique des dispositifs de formation / remédiation proposés dans les universités pour les publics non francophones. La discipline «français", où l'on apprend les règles de grammaire, d'orthographe, mais aussi les pratiques d'écriture, n'existe pas en tant que telle à l'université (Delcambre et Lahanier-Reuter, 2010), même si les ateliers ou dispositifs de formation du type «techniques d'expression »s'en rapprochent. Notre pratique de l'enseignement auprès des publics universitaires non francophones nous conduit à nous interroger sur la part et la place de l'enseignement de la langue dans les dispositifs de formation à l'écrit universitaire à destination de ces publics, et sur la façon de concevoir l'apprentissage linguistique à l'intérieur du concept de littératie universitaire.

Considérant qu'il convient d'envisager la problématique de la maitrise de la langue en passant d'une approche purement linguistique à une approche discursive (Pollet, Glorieux et Toungouz, 2010 : 61), les questions que nous nous posons sont les suivantes :

- quelle place accorder aux compétences linguistiques dans les dispositifs de formation aux écrits universitaires, et en particulier, dans les dispositifs destinés aux étudiants / doctorants non francophones? quel type de dispositif de formation mettre en œuvre ?

- en particulier, comment sortir du «micro linguistique», tant dans la gestion des énoncés considérés comme déviants, que dans les remédiations proposées ?

Pour ce faire, nous observerons un dispositif de formation à l'écrit universitaire proposé à un groupe de huit doctorants algériens. Ces huit doctorants réalisaient des thèses en Sciences du Langage, sur des sujets divers (toponymie, analyse des discours publicitaires, journalistiques, etc.). Dans le cadre d'une collaboration entre universités française et algérienne, une demande de formation à l'écrit de recherche avait été faite par leurs universités d'origine, formation qui devait comporter des modules de méthodologie mais également de langue.

Le fait qu'il s'agisse de doctorants algériens inscrit la problématique de la langue dans un registre particulier. Le français ne peut en effet pas être considéré comme une langue étrangère pour ce type de public, mais plutôt comme une langue seconde. Or, leurs difficultés 
linguistiques sont réelles, même si elles sont très variables selon les individus et leurs parcours. Et le fait que ce public ne puisse pas être considéré comme non francophone fait qu'il bénéficie rarement d'une véritable prise en charge de ses difficultés linguistiques (contrairement aux publics issus d'autres pays).

La formation proposée, étalée sur deux semaines, comportait plusieurs modules de formation à l'écrit de recherche (présenter un travail de recherche/élaborer une proposition de communication, positionnement énonciatif et citation, recherche documentaire) dont un module intitulé «Aspects linguistiques et stylistiques des écrits de recherche ». En amont de la formation, les doctorants avaient fait parvenir aux formateurs un chapitre de leur thèse, soit environ 30 pages par doctorant. Ce module proposait, à partir d'un corpus d'incorrections linguistiques relevées dans les productions écrites des doctorants, une série d'activités didactiques centrées sur les difficultés observées. Ces activités suivaient une progression, partant d'exercices sur des points de grammaire ou de lexique (paronymie, pronoms) pour aller vers des activités portant sur l'organisation de la phrase (accord des verbes dans les phrases complexes, ponctuation, organisation des phrases subordonnées, etc.), puis vers des activités de réécriture (textes issus de blogs à l'écriture défaillante qu'il s'agissait de modifier) et d'écriture (rédaction d'une introduction et d'un court texte argumenté sur des sujets imposés).

A partir de ce dispositif de formation, l'observation que avons menée pour la présente recherche s'est déroulée comme suit. Nous avons tout d'abord observé les productions écrites des doctorants afin d'analyser et d'essayer de classifier les énoncés pouvant être considérés comme déviants. Ainsi, nous avons tenté d'observer quelles parties du discours étaient le plus souvent concernées; ensuite, nous avons essayé d'analyser un possible lien entre ces déficiences linguistiques et la compétence de production écrite dans son ensemble.

Nous nous sommes ensuite intéressée au dispositif de remédiation proposé : au-delà de la satisfaction immédiate des apprenants, toujours rassurés par un travail sur les habiletés linguistiques, ce type de dispositif permet-il une véritable montée en compétence en production écrite? L'objectif final de cette recherche est ainsi de mettre en place des pratiques pédagogiques, des dispositifs de formation, qui permettraient de favoriser le passage du linguistique au discursif.

Il convient de préciser que cette recherche est exploratoire, et que les analyses effectuées sont constamment remises en question et alimentées par de nouvelles expériences pédagogiques.

\section{Résultats}

\subsection{Types d'énoncés déviants}

Nous avons tout d'abord procédé à un relevé, dans les écrits de ces doctorants, des énoncés ou parties d'énoncés pouvant être considérés comme «déviants » d'un point de vue linguistique (même si, nous le verrons plus loin, la frontière entre le linguistique et le scientifique n'est pas facile, ni même légitime, à établir).

Il n'est d'écart que par rapport à une norme. Attendre d'apprenants non natifs une production «sans faute » n'est ni réaliste ni même souhaitable. Toutefois, rappelons qu'il s'agit ici de doctorants, et que la norme attendue est donc celle d'un texte rédigé dans une langue académique correcte. 
Figurent dans ce relevé des énoncés dont les degrés d'écart par rapport à la norme attendue sont très divers : fautes d'orthographe, erreurs de grammaire, imprécisions lexicales, etc. Nous avons établi une classification de ces énoncés déviants en fonction des parties du discours qui étaient concernées et du degré d'influence de ces erreurs sur la cohérence textuelle. Nous adoptons ici la classification des erreurs proposée par Marquillo-Larruy (2003 : 87). Le niveau infraphrastique englobe les questions d'orthographe et d'accentuation. Marquillo-Larruy y inclut également le choix du lexique, mais nous avons pour notre part classé les écarts quant au choix du lexique dans une catégorie à part. En effet, il nous semble que le choix d'un lexique approprié dépasse le seul niveau infraphrastique (voir les travaux de Cavalla, 2007 \& 2008). Le niveau phrastique concerne l'ordre des mots, la construction verbes/prépositions, la construction des phrases complexes. Le niveau transphrastique et textuel concerne la cohérence globale du texte, la progression de l'information, l'usage adéquat de la ponctuation, la cohérence des systèmes temporels. Marquillo-Larruy rajoute à cette trame d'observation des productions d'apprenants un niveau pragmatique, que nous n'avons pas pris en compte dans cette étude, le public et les productions observées ne s'inscrivant pas dans la même perspective (l'ouvrage cité propose d'observer des productions d'apprenants répondant à des consignes) ${ }^{2}$.

Nous pouvons alors proposer le schéma suivant, dans lequel le degré d'influence de ces erreurs sur la cohérence textuelle est représenté par la flèche verticale :

Erreurs au niveau infraphrastique

Erreurs lexicales

Erreurs au niveau phrastique

Erreurs au niveau transphrastique et textuel

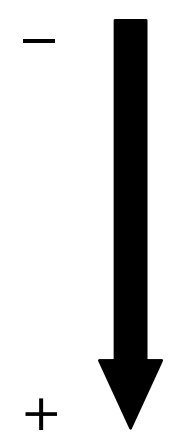

Dans un premier temps, nous pouvons considérer que les écarts qui ne concernent que les niveaux infraphrastiques ont une influence moindre sur la cohérence textuelle dans son ensemble que des déficiences au niveau de la construction de la phrase ou du texte. Quant aux erreurs concernant l'utilisation du lexique, nous le verrons dans les exemples ci-dessous, elles affectent essentiellement la précision du message.

Marquillo-Larruy (2003: 93) parle de démarche «descendante» dans l'analyse de productions d'apprenants, démarche qui va des aspects globaux du texte vers les niveaux qui traitent des composants premiers (mots, orthographe, etc.). Nous avons pour notre part adopté une démarche "ascendante ", en nous attachant tout d'abord au niveau infraphrastique. Le schéma proposé ci-dessus constitue avant tout un outil de travail et peut être amené à évoluer. En effet, nous verrons qu'il est possible de considérer que la cohérence textuelle est compromise dès les premiers niveaux d'écart.

\footnotetext{
${ }^{2}$ On peut bien sûr considérer que tout genre a une visée pragmatique (expliquer ou démontrer, dans le cadre de la thèse, par exemple), mais le fait de n'avoir observé que des extraits, et non pas les thèses dans leur ensemble, nous a conduit à ne pas prendre en compte cet aspect.
} 
Nous allons à présent illustrer cette typologie par des exemples représentatifs des erreurs relevées dans les textes des doctorants.

\subsubsection{Erreurs au niveau infraphrastique}

Figurent dans cette catégories les erreurs concernant l'orthographe lexicale :

les outilles - une nourrisse - voir / voire

On relève aussi de nombreuses confusions dues à l'homophonie :

$\mathrm{er} /$ é - et / est - ce / se - c'est / s'est

On retrouve également de nombreux exemples concernant l'orthographe grammaticale, comme des problèmes d'accord des participes passés :

La discipline théorique qui a engendrée la grammaire transphrastique

Plusieurs noms de lieux de souche française ont été remplacé par les noms de certains martyrs

\subsubsection{Erreurs lexicales}

S'agissant de doctorants algériens, pour lesquels le français ne peut être considéré comme une langue «étrangère » (le français est en effet langue d'enseignement dans le supérieur), les erreurs dans le domaine de l'usage du lexique se rapprochent de celles que l'on pourrait rencontrer chez un public de natifs. Ces erreurs relèvent surtout de la paronymie, source de confusion, et du manque de précision dans le choix des unités lexicales.

- Exemples de confusion due à la paronymie :

Combien même cet aspect théorique du travail (...)

A coteaux tirés

mettre sur le devant de la seine

Cette initiative débauche quand même, en janvier 1992, sur le passage au numérique le souk est considéré au même temps comme un endroit pervers, plein de djinns et d'esprits malfaiteurs

- Exemples d'imprécision ou confusion lexicale :

Notre présente contribution à l'étude de la toponymie d'une partie de l'Algérie $s$ 'inscrira avant tout dans un ancrage linguistique pallié à d'autres disciplines

Nous tenterons de mener à bien notre étude en nous basons sur une approche inférentielle

3.1.3. Erreurs au niveau phrastique, transphrastique et textuel

Au niveau de la phrase, on retrouve de nombreux exemples d'erreurs d'accord des verbes ou des adjectifs :

Cette citation d'Albert Dauzat résume en quoi consiste les catégories toponymiques

On relève également de nombreux exemples d'usage défaillant des prépositions :

Rien ne préexiste le langage

La géographie est d'un atout considérable aux études onomastiques

Si l'on se situe maintenant au niveau transphrastique et textuel, on remarque tout d'abord un usage défaillant de la ponctuation, qui est soit mal utilisée (virgules mal placées, tirets mal employés, entre autres) soit insuffisante : 
La linguistique textuelle, quant-à-elle, est la branche de la linguistique qui se fixe en tant qu'objet d'étude la cohérence d'un texte ou d'un discours elle est située aux frontières entre la linguistique transphrastique et l'analyse du discours.

On peut également relever de nombreux exemples mettant en évidence des défaillances concernant l'anaphore, en particulier dans l'usage du pronom « en » et des pronoms relatifs :

De ces quatre axes, Kerbrat-Orecchioni n'en garde que les deux premiers

C'est un véritable phénomène de société qui change les mours plus que n'en font les discours religieux, philosophiques, politiques ou autres

Mais il ne faut pas oublier que les Algériens en raffolent des assiettes paraboliques depuis leur introduction dans le pays

Les consommateurs algériens du fait de cette acculturation dont nous avons évoqué (...)

La concordance des temps, et de façon générale l'accord des verbes dans les phrases complexes, sont aussi sources d'énoncés incorrects :

Nous allons voir dans les lignes qui suivent, qu'en Algérie les réformes dans le domaine étaient imminentes également, mais qui seront un peu entravées, à cause des bâtons que la bureaucratie lui mettait dans les roues

La publicité commerciale est-elle une véritable source de progrès ou bien une machine idéologique qui élèvent les uns et rabaissent les autres?

On peut aussi relever de nombreux énoncés présentant une syntaxe approximative, bien souvent des phrases trop longues dans lesquelles les différents moyens de reprise de l'information ne sont pas maîtrisés (voir exemples ci-dessus). L'exemple suivant est assez caractéristique :

La tradition veut que le terme de «texte» soit utilisé pour représenter des productions verbales qui sont décontextualisées, autrement dit, le texte est souvent considéré hors de son contexte de production et cela dans le but de permettre à cette structure généralement comportant des productions importantes et qui sont pour la plus part sensés durer, et circuler loin du contexte de la production.

Ce dernier exemple montre une accumulation de micro-énoncés déviants, auxquels s'ajoute une syntaxe assez défaillante, le tout aboutissant à un énoncé incohérent.

\subsection{Quel degré d'influence sur la cohérence textuelle ?}

Au vu des différents exemples cités, nous pouvons observer les faits suivants :

- Nous considérons que même en haut de l'échelle présentée ci-dessus, les compétences purement microlinguistiques (qui affectent le niveau infraphrastique), compromettent déjà la cohérence textuelle, ne serait-ce qu'au niveau pragmatique, par le fait que ces énoncés déviants ne respectent pas une norme attendue pour ce type d'écrit, qui est celle de produire un texte correct au niveau de la langue. De plus, les erreurs au niveau infraphrastiques sont rarement isolées, et l'on constate souvent, comme dans le dernier exemple ci-dessus, une accumulation de micro énoncés déviants qui compromettent toute la cohérence textuelle. Ainsi, un usage défaillant des prépositions et des pronoms de reprise gêne la progression thématique; il en va de même avec la ponctuation. Quant à la compétence lexicale, nous avons vu que l'imprécision dans le choix des termes compromet le sens du discours dans son ensemble. 
- On peut également s'interroger sur le lien cohérence textuelle/cohérence scientifique. En effet, on peut aisément remarquer que les textes les plus faibles au niveau linguistique sont aussi les plus faibles au niveau scientifique ${ }^{3}$. Même si cette corrélation est tout sauf surprenante, il serait intéressant de pouvoir analyser quelle est la «faiblesse » première ou prépondérante (mais une formation courte et ponctuelle ne permet pas un accompagnement sur le long terme de ce public et une analyse fine de leurs difficultés $\left.{ }^{4}\right)$. Est-ce parce que le scripteur de maîtrise pas le raisonnement scientifique que son expression est défaillante, ou le contraire ? S'il est clair que, dans le cas présent (rappelons qu'il s'agit de doctorants algériens), les deux sont étroitement corrélés (nous y reviendrons plus loin), les résultats d'une telle observation seraient peut-être très différents avec une population pour laquelle la langue française est très éloignée et n'est pas langue d'enseignement. Nous pensons en particulier aux publics asiatiques, très nombreux dans les filières de nos universités, notamment dans les filières scientifiques. Pour ce type de public, l'éloignement touche non seulement la langue, mais également le modèle de raisonnement scientifique, ainsi que les modes de transmission des connaissances. Comme nous l'avons souligné dans l'introduction, on ne situerait plus dans un processus de remédiation mais bien de perfectionnement et même d'acquisition de compétences linguistiques et méthodologiques.

\subsection{Dispositif de remédiation}

Intéressons-nous à présent au dispositif de remédiation proposé à ces doctorants. Il s'agissait d'un module de six heures, inclus dans un dispositif de formation d'un total de trente heures ; les autres modules portaient soit sur la langue (la phraséologie propre aux écrits scientifiques), soit sur la méthodologie de l'écrit de recherche (la proposition de communication, le positionnement énonciatif et la citation), soit encore sur la méthodologie de la recherche documentaire. Ce module de six heures portait explicitement sur les aspects linguistiques des écrits de recherche. Les doctorants participant à ce stage avaient fait parvenir aux formateurs un chapitre de leur thèse ; les formateurs avaient ainsi observé et analysé ces écrits, afin de procéder à un relevé et une typologie des énoncés déviants.

Le travail en classe a ensuite consisté en des exercices de langue portant sur les points défaillants apparaissant dans les énoncés considérés comme déviants (voir les exemples cités ci-dessus : lexique, ponctuation, pronoms compléments et relatifs, etc.). Ces exercices étaient soit issus de manuels de Français Langue Etrangère de niveau avancé, soit conçus par l'enseignant à partir de textes authentiques (productions des doctorants, textes littéraires ou journalistiques). Ces différents exercices de langue faisaient ensuite l'objet d'une application lors de la réécriture/écriture de phrases puis de textes. Les textes à produire s'inscrivaient dans la thématique du stage de formation, celles des écrits scientifiques universitaires (introduction de chapitre, résumé de communication, entre autres).

Nous pouvons donc remarquer que deux niveaux de compétences sont visés et exploités dans ce module : le niveau micro, avec un travail sur des points de langue, et le niveau méso, avec une application à l'écriture de textes courts. Mais il y manque un niveau macro, qui correspondrait à un travail sur la capacité de rédaction d'écrits longs, de type article ou thèse.

\footnotetext{
${ }^{3}$ S'agissant de doctorants en Sciences du Langage, le contenu scientifique nous était accessible, ce qui n'aurait pas été le cas avec des productions d'autres domaines, telles que les sciences dures, par exemple.

${ }^{4}$ De même, une telle étude impliquerait de travailler en étroite collaboration avec les directeurs de thèse, et donc de ne pas isoler le travail sur la langue.
} 
Il est clair qu'il est difficile de viser une telle compétence dans un module de formation court. De plus, d'un point de vue pédagogique, il est plus aisé de mettre en place des activités sur des habiletés microlinguistiques que sur la production d'écrits longs. Et c'est bien là, à notre avis, la principale limite de ce type de dispositif de remédiation. Si l'articulation entre les niveaux micro et meso est relativement aisée à mettre en évidence d'un point de vue pédagogique (l'écriture ou réécriture de textes courts permet de mettre en pratqiue le travail précédent sur les points de langue), l'articulation avec le niveau macro suppose un dispositif de formation concu de façon beaucouop plus globale.

Dans les aspects positifs de cette formation, nous pouvons relever le fait que ce module axé sur les compétences linguistiques n'était pas isolé mais faisait partie intégrante du stage dans son ensemble. Tous les formateurs avaient établi leur programme en étroite collaboration, et à partir des productions envoyées par les doctorants. De même, il convient de souligner que les activités proposées dans ce module ont rencontré un écho très favorable de la part des participants, qui ont eu le sentiment de combler des lacunes, et ont apprécié de travailler à partir de leurs propres productions. Toutefois, au-delà de la satisfaction immédiate des apprenants, on peut réellement s'interroger sur l'efficacité à long terme d'un tel dispositif. Ces quelques heures de formation consistaient en effet essentiellement en une remédiation ponctuelle portant sur des habiletés linguistiques, mais ont-elles permis une véritable montée en compétence en production écrite ? S'il est certes bien difficile de répondre à cette question, car une évaluation de l'évolution de ce type de compétence ne peut se faire que sur le long terme, nous croyons pouvoir affirmer que ce module a apporté des réponses concrètes et efficaces à certaines déficiences linguistiques, mais n'a pas permis un vrai travail de production écrite.

\section{Conclusions et perspectives}

La première et principale conclusion à laquelle nous arrivons, non seulement à l'issue de cette observation, mais du fait de notre expérience d'enseignement auprès des publics universitaires non francophones, ou plus précisément non natifs, c'est-à-dire les publics que nous qualifierons de FLE/FLS ${ }^{5}$, c'est qu'il y a effectivement une forte demande et des besoins avérés en formation linguistique sur objectif universitaire. Les dispositifs actuels, de formation ou de remédiation à destination de ces publics, ne permettent pas, la plupart du temps, un travail sur le long terme: ils consistent généralement en modules de formation/remédiation ponctuels et essentiellement «palliatifs ». En effet, les dispositifs de formation linguistique à destination de ces publics consistent le plus souvent en formations courtes, soit sous forme de stage intensif ou semi-intensif (cas du dispositif analysé dans cet article), ou de formations extensives sur un semestre, mais à raison de quelques heures hebdomadaires.

Ensuite, on constate le plus souvent un cloisonnement entre la prise en charge des difficultés linguistiques et celle de la compétence de production écrite dans les disciplines, en particulier dans les domaines scientifiques. Ainsi, la remédiation linguistique revient généralement aux Centres Universitaires de Français Langue Etrangère, ou aux départements de Sciences du Langage ou Langues des universités, alors que la compétence d'écrit disciplinaire reste attachée aux départements dans lesquels ces étudiants poursuivent leur cursus. On a parfois l'impression que les filières spécialisées demandent aux formations linguistiques de remédier

\footnotetext{
${ }^{5}$ Français Langue Etrangère / Français Langue Seconde
} 
à toutes les difficultés des étudiants non natifs, alors que parmi ces difficultés on identifie clairement des déficiences méthodologiques voire disciplinaires (Carras, 2006).

Comme nous l'avons dit plus haut, la présente analyse a été faite avec des doctorants algériens en Sciences du Langage, soit un public de FLS, de culture universitaire francophone, et suivant un cursus qui nous est familier. Notre intention est de renouveler l'expérience et de poursuivre cette étude avec des étudiants de cultures universitaires et scientifiques plus éloignées, et de niveau linguistique plus faible. En effet, ces étudiants, et nous pensons spécifiquement au public asiatique, connaissent des difficultés d'ordre linguistique mais également disciplinaire. Et les difficultés observées chez ce type de public plaident tout à fait en faveur d'un décloisonnement des compétences.

Quelles pistes pouvons-nous proposer à l'issue de cette étude ? Que faire pour améliorer la prise en compte des difficultés des publics non francophones face à l'écrit dans les disciplines?

La première proposition que nous pourrions faire, consiste à souligner la nécessité de décloisonner les compétences linguistiques et celle de production écrite dans les disciplines. La notion de "littératie universitaire » est, comme de nombreux auteurs l'ont écrit (entre autres, Delcambre et Lahanier-Reuter, 2010), essentiellement pluridisciplinaire. De plus, comme nous l'avons dit plus haut, il est clair que la compétence en langue, si elle est essentielle, n'est pas isolée ni «étanche » par rapport à la compétence disciplinaire. Par contre, ce décloisonnement va de pair, selon nous, avec une véritable reconnaissance de la part du linguistique dans cette compétence. Tout ceci peut paraître quelque peu contradictoire, mais lorsque nous parlons de reconnaissance de la part de la compétence linguistique dans la compétence disciplinaire dans son ensemble, nous pensons également à la reconnaissance institutionnelle. Ainsi, on pourrait espérer, de la part des filières intégrant des étudiants non natifs dont le niveau ne leur permet pas de suivre de façon satisfaisante leur cursus ${ }^{6}$, une mise en place de formations linguistiques sur le moyen, voire le long terme, et pas seulement de dispositifs de remédiations ponctuels. Cette reconnaissance institutionnelle, tout comme le décloisonnement des compétences, suppose une collaboration entre les enseignants de FLE et les enseignants de discipline, mais ceci est, jusqu'à présent, un vœu pieux ...

Pour terminer, un dernier exemple issu du corpus en faveur du décloisonnement :

Dans un second temps, il nous a paru également indispensable d'évoquer la publicité française, son histoire, bref, ses lois qui la régissent, avant de mettre sur le devant de la seine l'opérateur Orange qui, pour le moment, absorbe tous les opérateurs existants en France pour devenir la première marque mondiale dans le futur proche. Une ambition qui se fonde sur un management jugé des plus outranciers par les différents médias et les syndicats des travailleurs. De plus, le passage de la marque, d'un simple opérateur historique à celui d'acteur mondial ne s'est pas fait sans déclencher un nombre considérable de suicides chez ses fonctionnaires. Ceux-ci vont inciter les anti-pubs et les parodistes à donner du fil à retorde à la compagnie Orange. Nous allons réaliser que celle-ci ne se décourage pas et fait tout pour peaufiner son image.

Exemple dans lequel on peut observer un manque évident de cohérence scientifique et donc de cohérence textuelle, qui s'exprime par la langue, mais auquel l'enseignant de langue ne peut, seul, remédier ...

\footnotetext{
${ }^{6}$ Niveau insuffisant dont se plaignent régulièrement les enseignants de disciplines.
} 


\section{Bibliographie}

CARRAS, C., 2006, «Quelles réponses didactiques pour l'intégration des étudiants étrangers dans les cursus universitaires français? Un cas spécifique d'enseignement / apprentissage du FLE en milieu homoglotte », dans D. Abry, M. Fiévet (dir.), L'enseignement apprentissage $\mathrm{du}$ français langue étrangère en milieu homoglotte: spécificités et exigences, PUG, ADCUEFE, p. 33-45.

CARTON, F., 2002, «Discours des enseignants d'université sur la compétence en expression écrite de leurs étudiants », Enjeux, 53-54, p. 162-175

DEFAYS, J-M, ENGLEBERT, A. (dir.), 2009, Principes et typologie des discours universitaires / Acteurs et contextes des discours universitaires, Paris, L'Harmattan.

CAVALLA, C., 2007, «Réflexion pour l'aide à l'écrit universitaire auprès des étudiants étrangers entrant en Master et Doctorat », dans J. Goes, J.- M. Mangiante (dir.), L'accueil des étudiants étrangers dans les universités francophones : sélection, formation et évaluation, Arras, Artois Presses Université, p. 37-48.

CAVALLA, C., 2008, «Les collocations dans les écrits universitaires : un français spécifique pour les apprenants étrangers », dans O. Bertrand, I. Schaffner (dir.), Apprendre une langue de spécialité : enjeux culturels et linguistiques, Paris, Editions Ecole Polytechnique, p. 93104.

DELBART, A.-R., 2002, «Quelle norme pour l'écrit à l'université ? », Enjeux, 53-54, p. 153161.

DELCAMBRE, I., LAHANIER-REUTER, D., 2010, Les littéracies universitaires : influence des disciplines et $d u$ niveau d'étude dans les pratiques de l'écrit, http://forumlecture.ch/myUploadData/files/2010_3 Delcambre_Lahanier.pdf

DELCAMBRE, I., LAHANIER-REUTER, D. (dir.), 2012, Littéracies universitaires : nouvelles perspectives, Pratiques, 153-154, Metz, Cresef.

LABORDE-MILAA, I., BOCH, F., REUTER, Y. (dir.), 2004, Les écrits universitaires, Pratiques, 121-122, Metz, Cresef.

MANGIANTE, J-M et PARPETTE, C., 2010, Le Français sur Objectif Universitaire, Grenoble, PUG.

MARQUILLO LARRUY, M., 2003, L'interprétation de l'erreur, Paris, CLE International.

MORTAMET, C., 2002, «La maîtrise du français à l'entrée à l'université : une question d'hétérogénéité ou d'altérité ? », Enjeux, 53-54, p. 115-127.

POLLET, M.-C., 2001, Pour une didactique des discours universitaires, Bruxelles, De Boeck Université.POLLET, M-C, GLORIEUX, C., TOUNGOUZ, K., 2010, « Pour un continuum dans l'appropriation de la littéracie universitaire », Diptyque, 18, p. 61-92.

RONVEAUX, C., 2010, «Écrire dans l'écrit des disciplines. Synthèse », Diptyque, 18, p. 153-163. 